\title{
Poly(ethylene glycol)-poly(tetrahydrofuran)-poly(ethylene glycol) triblock copolymer: Synthesis, crystallization behavior and novel morphology
}

\author{
W. W. Fan, X. D. Fan, W. Tian", X. Q. Liao, W. B. Zhang, C. G. Mu, J. Kong \\ The Key Laboratory of Space Applied Physics and Chemistry, Ministry of Education and Shaanxi Key Laboratory of \\ Macromolecular Science and Technology, School of Science, Northwestern Polytechnical University, Xi'an, 710072, P. R. \\ China
}

Received 15 November 2012; accepted in revised form 26 January 2013

\begin{abstract}
Poly(ethylene glycol)-poly(tetrahydrofuran)-poly(ethylene glycol) (PEG-PTHF-PEG) triblock copolymer was synthesized by ring-opening polymerization of ethylene oxide using sodium alcoholate of PTHF as the macroinitiator. Its crystallization behavior and formation mechanisms of different crystal structures were studied. The study showed that the molecular weight of PEG-PTHF-PEG exhibited a significant effect on its crystallization: that is, with the increase of the copolymer's molecular weight, the crystallizability of PTHF blocks decreased gradually, which led to the transition of copolymer from crystalline-crystalline to crystalline-amorphous. By adjusting the total molecular weight of triblock copolymer, the crystallization process can be effectively controlled, and as a result, different spherulite structures were obtained. Particularly, when PTHF blocks became amorphous, novel double concentric spherulites were observed. The morphological structures were studied by differential scanning calorimetry (DSC), Fourier transform infrared spectroscopy (FTIR), scanning electron microscope (SEM), polarized optical microscopy (POM), and its crystalline process was investigated.
\end{abstract}

Keywords: polymer synthesis, molecular engineering, crystallization, double concentric spherulites

\section{Introduction}

With the rapid development of polymer synthesis strategy, various block copolymers with desired structures were obtained, and at the same time, the crystallization behavior for these copolymers was investigated accordingly [1-5]. Block copolymers, consisting of both crystalline and amorphous segments were widely recognized that their crystallization processes and the resulting crystal morphologies can be significantly influenced by microphase separation in melt $[6,7]$. However, block copolymers, consisting of different crystalline segments such as the double crystalline block copolymers, usually exhibit much more complicated crystalliza- tion behaviors $[8,9]$, including confined crystallization [10-13], competitive or interactive crystallization and so on [14-16]. For these block copolymers, the overall crystallization behavior is influenced by block ratios, nucleation types, crystallization kinetics and characteristics of the individually folded chains [5, 17-19]. Recently, Li et al. [20] reported that the order of block crystallization in double crystalline block copolymers could be switched by adjusting total molecular weight, even while holding the block length ratio fixed. Their work revealed a convenient method to regulate the crystallization for double crystalline block copolymers, with the advantage that the block ratio or the content of each

\footnotetext{
${ }^{*}$ Corresponding author, e-mail: happytw_3000@163.com

(C) BME-PT
} 
component could be maintained. However, for the various block copolymers with different structures and components, the molecular weight dependence of their crystallization behavior should be diverse and much more complicated. Unfortunately, reports on this field are still inadequate till now.

Recently, with the comprehensive investigations on crystallization process for block copolymers, the high order structures containing crystal lamellae were frequently discovered [6, 21-25], where several types of spherulites with unusual morphologies such as ring banded spherulite, spherical granular aggregates and double concentric spherulites have been reported [14, 15, 26-31]. Especially, the double concentric spherulites were only found in poly(ethylene oxide)/poly( $\varepsilon$-caprolactone) PEG/PCL block copolymer up to now [14, 26-29]. This complex morphology contains an initial spherulite in center and a concentric outside spherulite with different crystal structure. The inner and outer spherulites are templated by crystallization of different blocks. Lately, Shi et al. [32] also reported a similar morphology in their study on PEG/PMMA blend and the formation was due to the different orientations of PEG crystal lamellae caused by the intervention of phase separation during crystallization process. Considering that the polymer's phase separation can also significantly affect the crystallization process of crystalline-amorphous block copolymers, double concentric spherulites may be expected to exist in crystalline-amorphous block copolymers. However, related studies were rarely reported up to now.

In this work, we synthesized several poly(ethylene glycol)-poly(tetrahydrofuran)-poly(ethylene glycol) (PEG-PTHF-PEG) as model triblock copolymers, where the copolymer's block ratios are equal and fixed, but their molecular weights are different. We focused our attention on controlling the copolymer's crystallization behavior via adjusting total molecular weight with a fixed block ratio to understand the detailed mechanism of the formation of crystalline structures for these triblock copolymers. The copolymer's crystallization process was monitored by DSC and their crystal morphologies were inspected via POM. The research data confirmed that the copolymer's molecular weight was a key factor to affect its crystallization behavior. Especially, when the molecular weight of triblock copolymer reached a certain value, the PTHF block became completely amorphous. Meanwhile the interesting double con- centric spherulites in this triblock copolymer were found. As far as we known, it was the first time that double concentric spherulites had been observed in crystalline-amorphous block copolymer. Our study could provoke more considerations in regulating the crystallization of block copolymers and may also be helpful for the design of crystalline materials.

\section{Experimental section}

\subsection{Materials}

Tetrahydrofuran (THF, Alfa Aesar, USA) was refluxed over Sodium (Na, 98\%, Sinopharm Chemical Reagent Co., Ltd, China) and freshly distilled before use. Ethylene oxide (EO, 98.0\%) was purchased from Sinopharm Chemical Reagent Co., Ltd (China) and stored with $3 \AA$ molecular sieve in sealed preserving condition. Sodium hydride (57$63 \%$ oil dispersion, Alfa Aesar, USA) was washed by anhydrous THF three times. Ethanol (99.5\%, Sinopharm Chemical Reagent Co., Ltd, China), perchloric acid (70 72\%, Sinopharm Chemical Reagent Co., Ltd, China), dichloromethane (99.5\%, Sinopharm Chemical Reagent Co., Ltd, China), nhexane (99.5\%, Sinopharm Chemical Reagent Co., Ltd, China), Amberlite IR-120(+) resin (Alfa Aesar, USA) and acetic anhydride (98.5\%, Sinopharm Chemical Reagent Co., Ltd, China) were all used as received.

Two types of Poly(ethylene glycol), denoted as PEG2000 and PEG8000, were purchased from Sinopharm Chemical Reagent Co., Ltd (China). Three types of hydroxyl-ended PTHF, denoted as PTHF1600, PTHF2600 and PTHF3900 (numbers stand for weight average molecular weights and the below is same), were purchased from Sigma (USA). For removing the residual moisture, the polymers were first dissolved in toluene and then distilled. The collected product was dried under the vacuum until a constant weight was obtained. The polymer's molecular weights were determined by size exclusion chromatography with multi-angle laser lights (SEC-MALLS).

\subsection{Synthesis of triblock copolymers (PEG-PTHF-PEG)}

Triblock copolymers with different molecular weights were synthesized by ring-opening polymerization of ethylene oxide using sodium alcoholate of PTHF as the macroinitiator. Preparation was car- 
Table 1. Molecular weight and composition of polymers

\begin{tabular}{|l|c|c|c|c|c|}
\hline \multicolumn{1}{|c|}{ Sample $^{\mathbf{a}}$} & $\mathbf{M}_{\mathbf{w}} \mathbf{b}^{\mathbf{b}}$ & $\mathbf{M}_{\mathbf{w}} / \mathbf{M}_{\mathbf{n}} \mathbf{b}^{\mathbf{b}}$ & $\mathbf{M n b}$ & $\mathbf{M}_{\mathbf{n}}{ }^{\mathbf{c}}$ & $\begin{array}{c}\text { PTHF in } \\
\mathbf{c o p o l y m e r}^{\mathbf{c}} \\
{[\mathbf{w t \%} \mathbf{0}}\end{array}$ \\
\hline PTHF1600 & 1660 & 1.25 & 1330 & 972 & 100 \\
\hline PTHF2600 & 2590 & 1.21 & 2140 & 1349 & 100 \\
\hline PTHF3900 & 3960 & 1.20 & 3300 & 2928 & 100 \\
\hline Block2950 & 2950 & 1.11 & 2660 & - & 48.6 \\
\hline Block4900 & 4910 & 1.09 & 4500 & - & 47.1 \\
\hline Block7800 & 7810 & 1.08 & 7200 & - & 47.8 \\
\hline
\end{tabular}

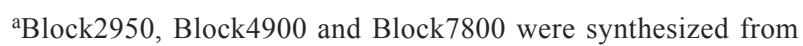
PTHF1600, PTHF2600 and PTHF3900 respectively.

${ }^{b}$ Determined by GPC-MALLS.

'PEG/ PTHF weight ratio in the block copolymer was determined from the area ratio of the ${ }^{1} \mathrm{H}$ NMR peaks at $3.64 \mathrm{ppm}$ (due to the PEG blocks) and $1.62 \mathrm{ppm}$ (due to the PTHF block). Then the content of PTHF in copolymer could be calculated.

ried out in an autoclave $(300 \mathrm{~mL}$, parr4566, Parr Instrument Company, USA), specifically, for Block7800, 40.07 g PTHF3900 was first dissolved in $50 \mathrm{~mL}$ of anhydrous THF, and then transferred into the autoclave. At the same time, $1.11 \mathrm{~g}$ of $\mathrm{NaH}$ was added and the air in autoclave was displaced subsequently by nitrogen. The temperature was raised to $60^{\circ} \mathrm{C}$ and retained for $3 \mathrm{~h}$ to make sure that $\mathrm{NaH}$ reacts with PTHF completely. After the reaction, the autoclave was cooled down in ice-water bath, and the atmosphere inside was replaced by nitrogen again. By that time, $48.00 \mathrm{~g}$ EO was added in, the temperature was increased to $75^{\circ} \mathrm{C}$ under stirring with a speed of $80 \mathrm{r} / \mathrm{min}$ for $12 \mathrm{~h}$. The polymerization was terminated by addition of $20 \mathrm{~g}$ of Amberlite IR-120(+) resin and stirred for another 6 hours. The final product was obtained by filtration from the resin into n-hexane $(500 \mathrm{~mL})$ at $0^{\circ} \mathrm{C} .{ }^{1} \mathrm{H}$ NMR (300 MHz, $\delta \mathrm{ppm}, \mathrm{CDCl}_{3}$ ): 3.64 (b, $\mathrm{OCH}_{2} \mathrm{CH}_{2} \mathrm{O}$ ), 3.41 (b, $\left.\mathrm{OCH}_{2} \mathrm{CH}_{2} \mathrm{CH}_{2} \mathrm{CH}_{2} \mathrm{O}\right), 1.62$ (b, $\mathrm{OCH}_{2} \mathrm{CH}_{2} \mathrm{CH}_{2} \mathrm{CH}_{2} \mathrm{O}$ ).

Two other block copolymers, denoted as Block2950 and Block4900, were synthesized following the similar procedure. The polymer's characteristics parameters were listed in Table 1.

\subsection{Characterization}

Molecular weights and polydispersity indexes of three triblock copolymers were determined by SECMALLS (DAWN EOS, Wyatt Technology Corporation, USA) equipped with a highly cross-linked styrene/divinylbenzene gel column (500 $\AA, 5 \mu \mathrm{m})$. HPLC grade THF was used as the eluent with a flow rate of $0.5 \mathrm{~mL} / \mathrm{min}$ at $25^{\circ} \mathrm{C}$. Samples were first diluted with THF to a concentration of ca. $5 \mathrm{wt} \%$ and then filtered through a filter of $0.22 \mu \mathrm{m}$. The refractive index increment $(\mathrm{d} n / \mathrm{d} c)$ value of samples was obtained by an Optilab rEX detector at $25^{\circ} \mathrm{C}$ through a batch model.

Structural compositions of the PEG-PTHF-PEG triblock copolymers were determined by ${ }^{1} \mathrm{H}$ NMR and ${ }^{13} \mathrm{C}$ NMR on a Bruker $300 \mathrm{MHz}$ spectrometer (Bruker Corporation, Germany) with DMSO as solvent and tetramethylsilane as the internal standard.

\subsection{Thermal and morphological characterization}

Differential scanning calorimetry (DSC) measurement was conducted on a TA 2910 instrument (TA Instruments, USA) calibrated with indium. The sample (3 5 mg) treated by freeze drying was encapsulated in aluminum pan and characterized with a heating rate of $5^{\circ} \mathrm{C} / \mathrm{min}$. Polarized optical microscopy $(\mathrm{POM})$ inspection was performed with a Nikon E400 optical microscope (Nikon Corporation, Japan) equipped with crossed polarizers $(\lambda=546 \mathrm{~nm})$ and a camera system. Fourier transform infrared spectroscopy (FTIR) was utilized to measure the characteristic vibrational bands of crystalline structures on a Nicolet iS10 instrument (Nicolet Instrument Corporation, USA). The samples for POM measurements were prepared by casting three drops of a $5 \mathrm{wt} \%$ tetrahydrofuran solution of the copolymer on a clean cover glass and then dried in a vacuum oven for $24 \mathrm{~h}$ at room temperature. $\mathrm{KBr}$ plate coated with sample was used for FTIR sample preparation. All samples before measurements were melted at $80^{\circ} \mathrm{C}$ for $10 \mathrm{~min}$ and then maintained at the crystallization temperature for $48 \mathrm{~h}$. For in situ POM and timedependent FTIR measurements, the samples were melted at $80^{\circ} \mathrm{C}$ for $10 \mathrm{~min}$ and then characterized immediately.

\section{Results and discussion}

\subsection{Synthesis of triblock copolymers}

According to the literatures, there are mainly two approaches to prepare PEG/PTHF block copolymers. One is to utilize chemical reaction to couple the hydroxyl-terminated PEO and PTHF segments [3335 ], and the other one is to terminate the cationic polymerization of THF by methoxypolyethylene glycols $[36,37]$. However, none of them can be regarded as the ideal synthetic strategies to prepare a qualified copolymer, typically, with a well-defined 
structure. Because the first method has a limited efficiency for coupling reaction and is time-consuming, and the latter requires a critically equal feed ratio, and frequently, the target block copolymer is often end-capped with methyl group. For this reason, we used anionic ring opening polymerization to prepare PEG-PTHF-PEG triblock copolymers, where hydroxyl-terminated PTHF carrying sodium alcoholate was used as the macroinitiator. As can be seen in Figure 1, the ionization of the terminal hydroxyl groups in PTHF can be accomplished via addition of sodium hydride, however, the ionized hydroxyls in PTHF should be lower than 50\% for preventing initiator precipitation from THF. It was found that as the exchange reaction between alcoholate active center and hydroxyl group is much faster than the initiation rate, which leads to simultaneous growth of polymer chains [38, 39]; therefore, accurate feeding of $\mathrm{NaH}$ is not necessary and as a result, the synthesis process becomes much easier to operate.

Using our synthetic approach, three triblock copolymers were prepared and their molecular weights and polydispersity indexes were listed in Table 1. The results of ${ }^{13} \mathrm{C}$ NMR measurements, as shown in Figure 2, also proved that the copolymer's structures are correct. The signals at $29.76 \mathrm{ppm}$ which can be assigned as the carbon of methylene-ended group in PTHF disappeared completely in PEG-PTHFPEG triblock copolymer, indicating all the PTHF being linked with PEG segment successfully.

Additionally, it should be pointed out that the polymerization is technically demanding. All solvents and reagents must be rigorously dried and degassed, and the purity of the sodium hydride is critical. In the synthesis, a possible problem is that $\mathrm{NaOH}$, which is formed from reaction of $\mathrm{NaH}$ with water, can induce the polymerization of EO and result in homopolymers. To get a deep scope, we added $0.5 \mathrm{wt} \%$ of water into EO during polymer synthesis. The final product was contaminated by PEG diol, and had a lower molecular weight as well as a broader
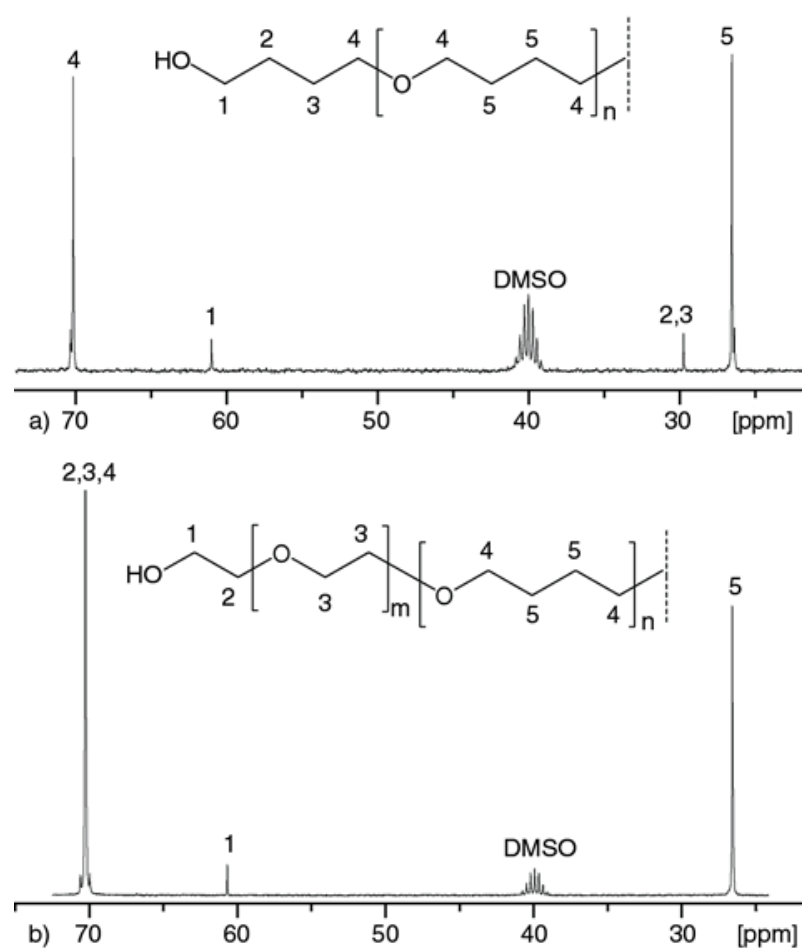

Figure 2. ${ }^{13} \mathrm{C}$ NMR spectrum of PTHF2600 (a) and Block4900 (b)

molecular weight distribution. Especially, an additional peak of PEG diol emerged in SEC elution curve, as shown in Figure 3b. With the strict control of processing conditions to avoid the contamination of moisture, triblock copolymer with desired molecular weight and narrow molecular weight distribution could be obtained, as shown in Figure 3c. Evidently, the peaks of PEG contaminant in SEC elution curve vanished. On the other hand, though the molecular weight of triblock copolymer could not be calculated by ${ }^{1} \mathrm{H}$ NMR spectra as the chemical shift of terminal groups was covered, the block ratio was easy to calculate and the value agreed well with that calculated from the number average molecular weight determined by SEC-MALLS, suggesting that the triblock copolymer was successfully prepared with high purity, which is very critical for the next crystallization behavior study.

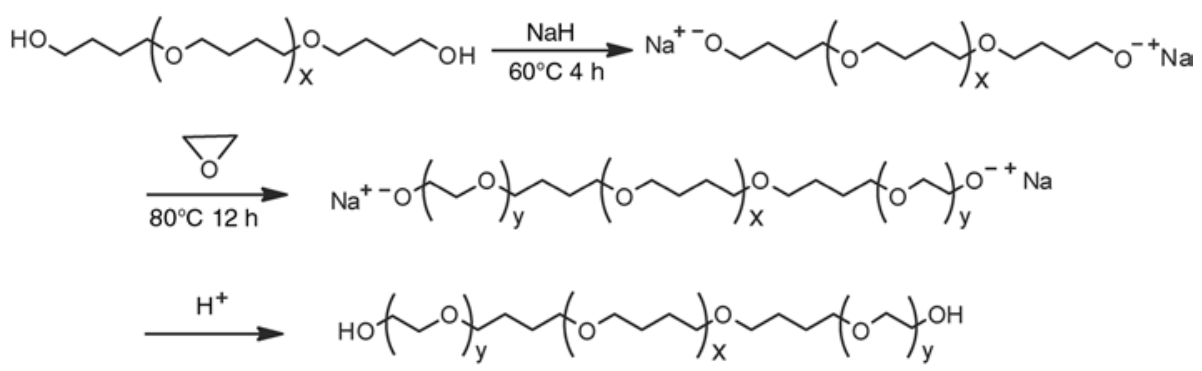

Figure 1. Synthesis route of triblock copolymers 


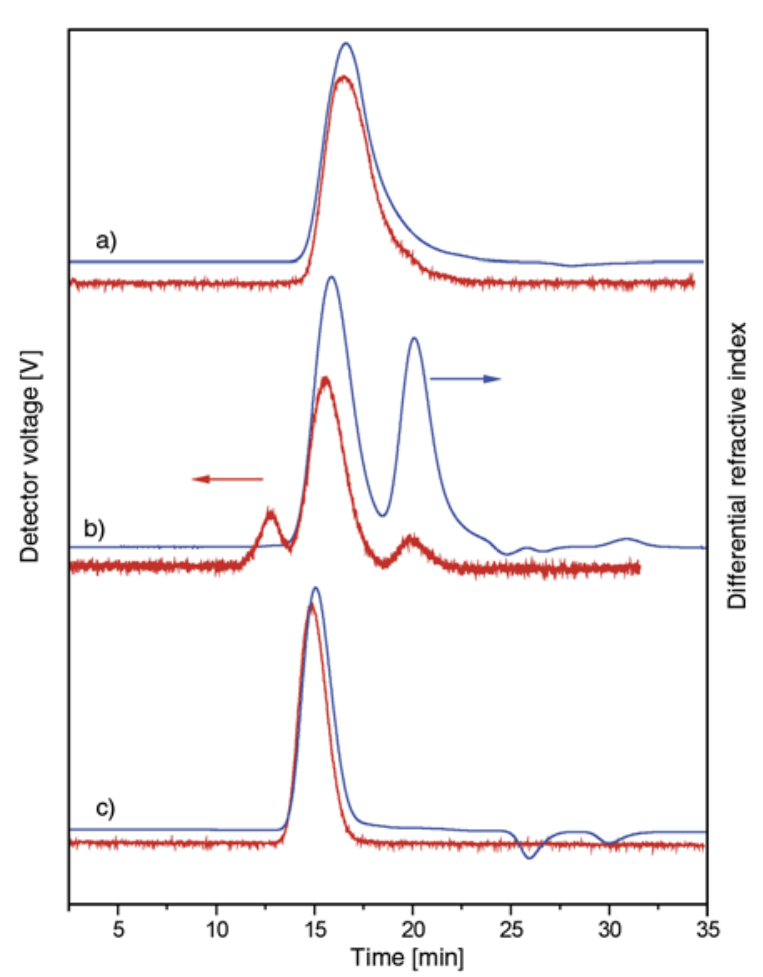

Figure 3. MALLS-SEC elution curves of polymers: (a) PTHF3900; (b) block copolymer synthesized with moisture in monomers; (c) Block 7800

\subsection{Relationship between crystallization and molecular weight}

The melting and crystallization behaviors of the PEG-PTHF-PEG triblock copolymers were investi-
Table 2. The $T_{\mathrm{m}}$ and $T_{\mathrm{c}}$ of the triblock copolymers

\begin{tabular}{|c|c|c|c|c|}
\hline \multirow[t]{2}{*}{ Sample } & \multicolumn{2}{|c|}{$\begin{array}{c}\text { Cooling } \\
\mathrm{T}_{\mathrm{c}}\left[{ }^{\circ} \mathrm{C}\right]\end{array}$} & \multicolumn{2}{|c|}{$\begin{array}{l}\text { Heating } \\
\mathrm{T}_{\mathrm{m}}\left[{ }^{\circ} \mathrm{C}\right]\end{array}$} \\
\hline & PTHF & PEG & PTHF & PEG \\
\hline PTHF1600 & 5.7 & - & 21.8 & - \\
\hline Block2950 & -3.3 & 7.1 & 18.6 & 25.6 \\
\hline Block4900 & \multicolumn{2}{|c|}{$7.4^{\mathrm{a}}$} & 23.5 & 33.3 \\
\hline Block7800 & - & 16.6 & - & 45.0 \\
\hline PEG2000 & - & 35.7 & - & 54.3 \\
\hline
\end{tabular}

${ }^{\mathrm{a} O v e r l a p p i n g ~ p e a k s ~}$

gated by DSC. Homopolymers of PEG2000 and PTHF1600 were also tested for comparison. Figure 4 shows the DSC curves collected from the measurements. The melting temperature $\left(T_{\mathrm{m}}\right)$ and crystallization temperature $\left(T_{\mathrm{c}}\right)$ are listed in Table 2.

As can be seen from Figure 4, both PEG and PTHF homopolymers are crystalline, and exhibited a fine single peak during crystallization and melting processes. In addition, the $T_{\mathrm{m}}$ of PTHF is much lower than that of PEG with the similar molecular weight, which is consistent with the literature [37]. Compared with the homopolymers, Block2950 exhibits two exothermic and two endothermic peaks. Both exothermic and endothermic peaks are rather lower than those of the homopolymers. That is because crystallization of one block significantly affects the crystallization behavior of the other block, and the crystallinity of both blocks tend to

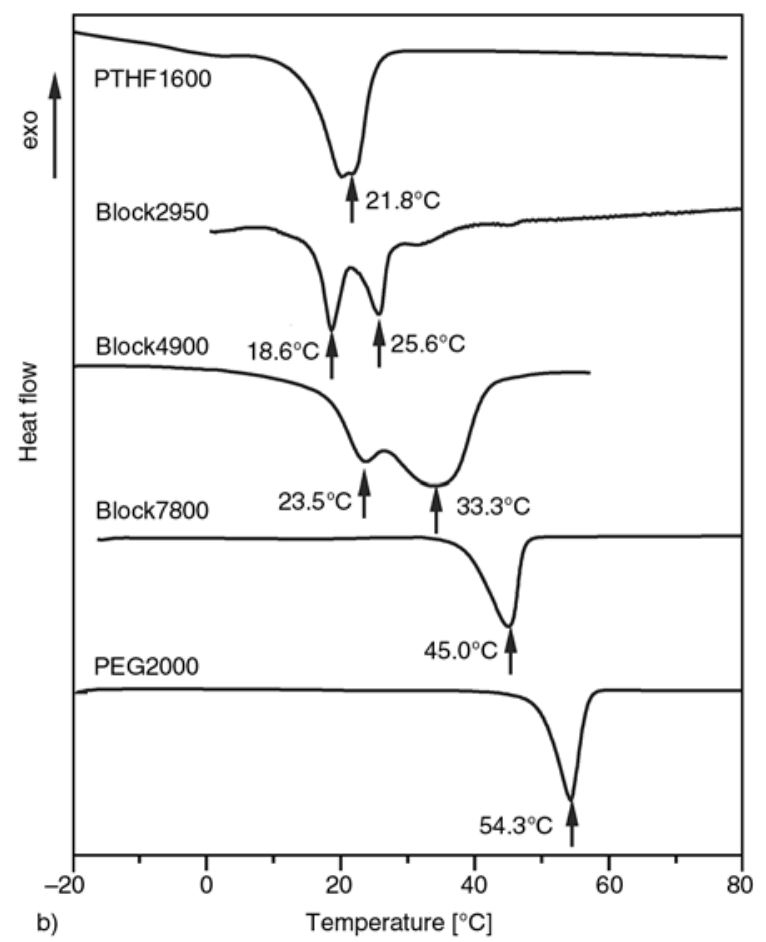

Figure 4. DSC cooling scans (a) and heating scans (b) at $5^{\circ} \mathrm{C} / \mathrm{min}$ after melting at $80^{\circ} \mathrm{C}$ for the indicated polymers 
decrease with the composition of the other block [5]. The peaks at lower temperatures can be assigned to the crystallization or crystalline melting of PTHF blocks, and the peaks at higher temperatures can be assigned to PEG block's contribution. However, the crystallization and melting temperatures for PTHF blocks are 9 and $3.2^{\circ} \mathrm{C}$ below that of PTHF 1600 , respectively. As the molecular weight in both homoand copolymers is basically the same, the result may indicate that the nucleation of the PTHF blocks in the copolymer was hindered by the PEG lamellae formed earlier, which are covalently bonded to them.

Following the increase in molecular weight, Block 4900 exhibits a single exothermic peak at $7.4^{\circ} \mathrm{C}$ but two endothermic peaks at 23.5 and $33.3^{\circ} \mathrm{C}$ respectively. From our FTIR results (discussed below), both PEG and PTHF blocks proved to be crystallizable, so the single exothermic peak may come from the overlap of the DSC signals during cooling. By the use of slower cooling rates, this overlap effect still cannot be overcome even at a cooling rate of $0.5^{\circ} \mathrm{C} / \mathrm{min}$, where a clear tailing area of crystallization was observed as shown in Figure 5. This may be related to the fact that, once the crystallization of PEG blocks started, it was quickly followed by the crystallization of the PTHF block which can be nucleated by PEG blocks, and this phenomenon was also reported in the literature by Müller et al. [40]. Besides, the intensity of endothermic peak of PTHF block was much weaker than that of PEG

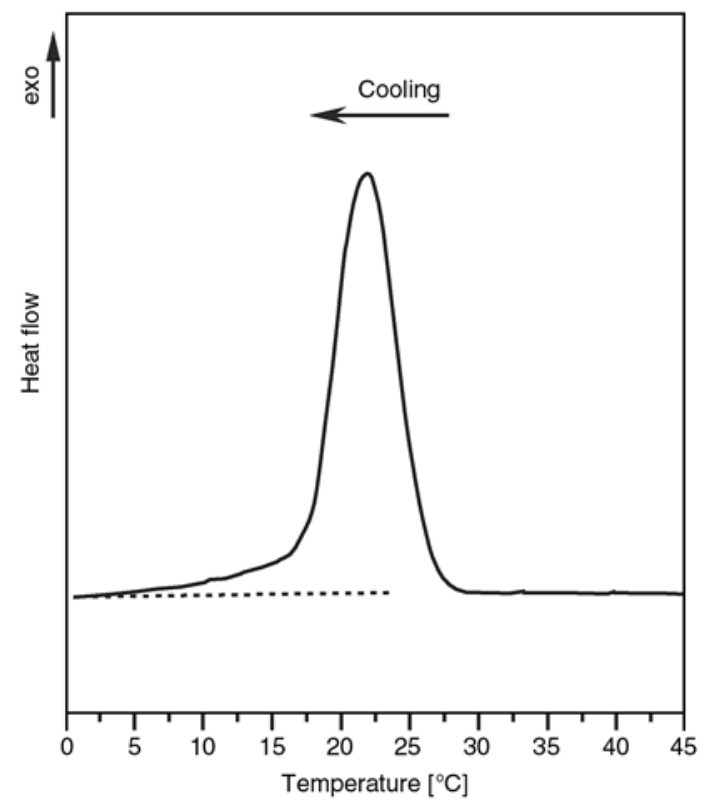

Figure 5. DSC cooling scan of Block 4900 at $0.5^{\circ} \mathrm{C} / \mathrm{min}$ blocks in Block4900, suggesting that the crystallizability of PTHF blocks was reduced following the increase in the molecular weight.

When the molecular weight increased further to 7800 , Block 7800 shows a single peak for both endothermic and exothermic processes. The melting point was also beyond the melting temperature range of homo-PTHF as reported before [37]. Therefore, it was reasonable to attribute the endothermic and exothermic peaks to PEG blocks only, and PTHF blocks may not be crystallizable in this molecular weight range. The conclusion was further confirmed by our FTIR data below. Additionally, it is worthwhile to note that, whether for PTHF or PEG blocks, the melting point and crystallization temperature were lower than that of the homopolymers with the same molecular weight. That is because the crystallization of PTHF and PEG segments in the block copolymers were restricted by each other. In the case of Block7800, even the PTHF blocks were noncrystallizable, the covalently bonded molten PTHF segments could also hinder the crystallization process of PEG blocks.

In order to further study the crystallization behavior, FTIR spectroscopy was used to monitor the crystallization process. The absorption band at $843 \mathrm{~cm}^{-1}$ in FTIR spectrum could be used to characterize the crystallization of PEG segments [27, 28]. On the other hand, characteristic absorption at $564 \mathrm{~cm}^{-1}$ for homo-PTHF crystals was also obtained by timedependent FTIR measurements during crystallization (Figure 6a). The intensity of absorption at $746 \mathrm{~cm}^{-1}$ could also characterize the crystallization of PTHF, but it should be noted that this absorption was inherent in PTHF even if it is totally amorphous. For triblock copolymers that crystallized at $-30^{\circ} \mathrm{C}$, as shown in Figure $6 \mathrm{~b}$, following the increase in molecular weight, the absorption intensities at 746 and $564 \mathrm{~cm}^{-1}$ gradually decreased implying the deduction of PTHF block's crystallization ability. Particularly, the disappearance of absorption band at $564 \mathrm{~cm}^{-1}$ for Block7800 demonstrates strongly that the PTHF blocks were totally amorphous within this copolymer. The result is in agreement with the data of DSC.

The change of crystallization behavior of block copolymers should be due to the different molecular weight dependence of crystallization of PEG and PTHF blocks, respectively. As molecular weight increased, the melting point of PEG blocks increased 

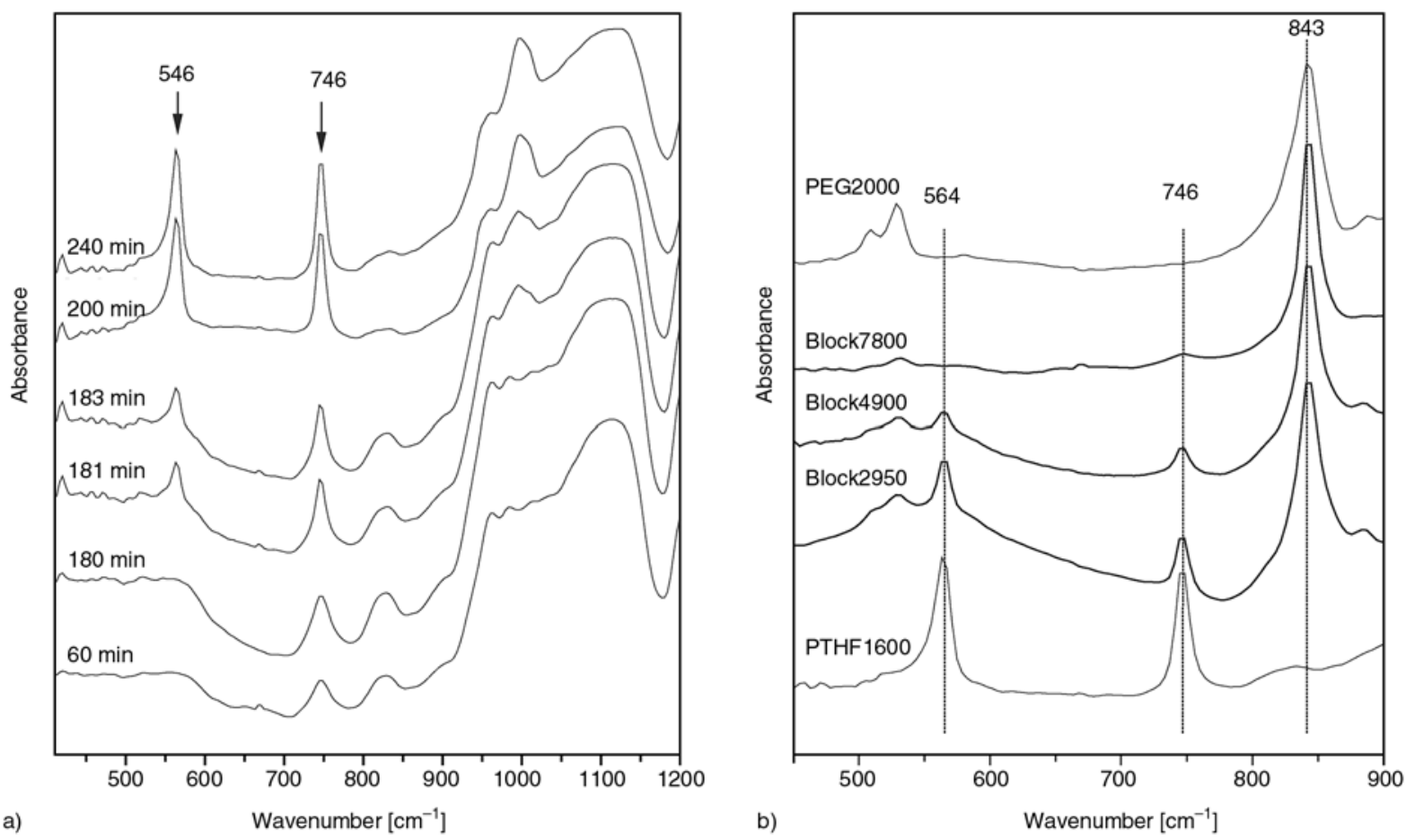

Figure 6. Time-dependent FTIR spectra (a) of PTHF2600 and FTIR spectra (b) for the indicated polymers

more rapidly than that of PTHF blocks, resulting in the further separation of melting temperatures of the two blocks (Figure 4). In general, when the melting temperatures of both blocks are near enough, a coincident crystallization phenomenon of both blocks can be obtained. Conversely, when the melting temperature of one block is far from the other, the latter crystallized component undergoes a confined crystallization process [5]. So the crystallization of PTHF blocks was steadily confined by PEG blocks as molecular weight increased, even causing PTHF blocks to be amorphous.

\subsection{Spherulite morphology}

For inspection of triblock copolymer's crystalline morphology changing with the molecular weight, the POM was employed to obtain micrographs of spherulites as shown in Figure 7. Obviously, the spherulites formed in Block2950 and Block4900 are closely packed with a broad size distribution. Similar phenomenon was observed in crystallization of PEG homopolymer, which may be attributed to different particle size fractions and the change of heat transfer under fast cooling conditions [41]. These spherulites exhibit negative Maltese crosses patterns, suggesting that the highest refractive index in the spherulites was tangential and coincided with the chain direction [27, 42]. Clearly, the amorphous domains were intercalated in the spherulites leading to minor color aberration of birefringent pattern and fibrillar crystals grow in the radial direction with short branches.

Following the increase in the molecular weight to 7800 , the numbers of spherulites were obviously reduced. Upon deepening quench to $-30^{\circ} \mathrm{C}$, fewer loose negative spherulites with much smaller size can be observed (Figure 7c). After carefully examining Figure 7c, it was found that many lamellar stacks were formed around the spherulites and seemed to orient randomly. Interestingly, when the crystallization temperature changed from -30 to $4^{\circ} \mathrm{C}$, the original spherulites kept a negative pattern but the lamellar outside oriented to form Maltese crosses with positive pattern (Figure 7d). As a result, the spherulites morphology of double concentric spherulites was observed, which was similar with the report of double crystalline PEG/PCL block copolymer [26-28].

\subsection{Formation of double concentric spherulites}

According to our study, crystallization process of Block7800 was able to lead to double concentric spherulites with $T_{\mathrm{c}}$ in range from 4 to $25^{\circ} \mathrm{C}$ or wider. The formation process of double concentric spherulites was studied by POM during isothermal crystallization at $22^{\circ} \mathrm{C}$. The real-time micrographs 


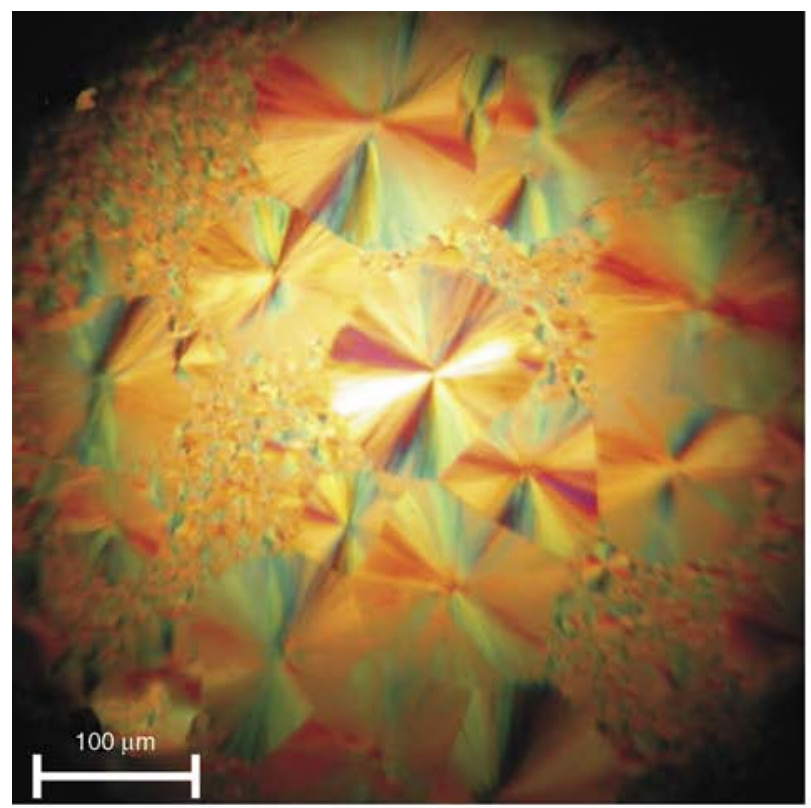

a)

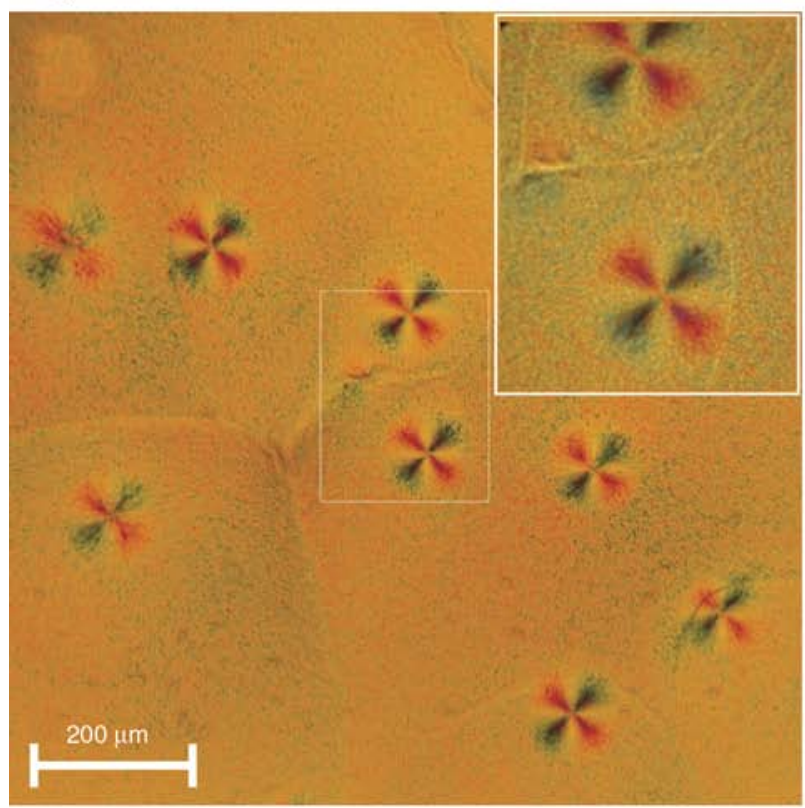

c)

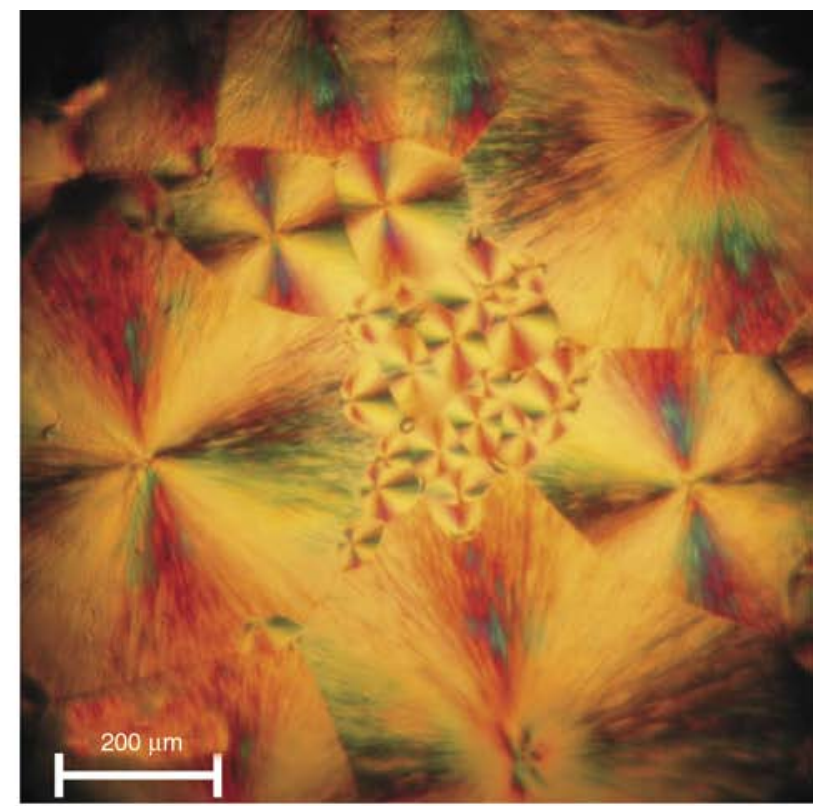

b)

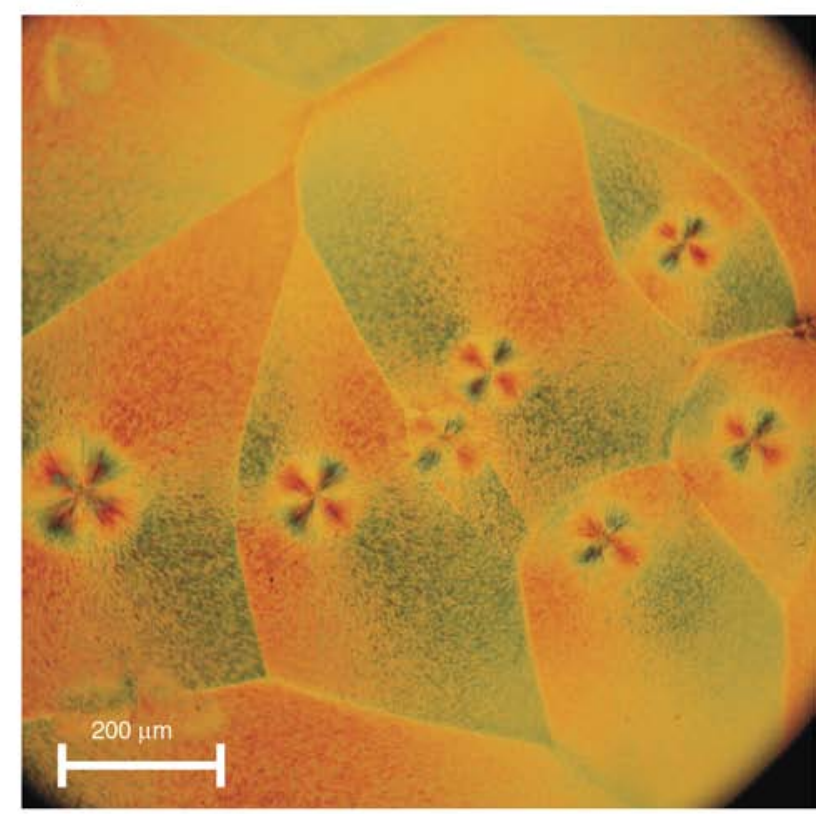

d)

Figure 7. POM micrographs: (a) Block2950, $T_{\mathrm{c}}=-30^{\circ} \mathrm{C}$; (b) Block $4900, T_{\mathrm{c}}=-30^{\circ} \mathrm{C}$; (c) Block $7800, T_{\mathrm{c}}=-30^{\circ} \mathrm{C}$; (d) Block $7800, T_{\mathrm{c}}=4^{\circ} \mathrm{C}$. All samples were melted at $80^{\circ} \mathrm{C}$ for $10 \mathrm{~min}$ and then transferred into thermostat to crystallize for $48 \mathrm{~h}$.

of Block7800 are presented in Figure 8. As shown, there are two spherulites with double concentric birefringence patterns formed in sequence, signed as spherulites 1 and spherulite 2 respectively. The formation processes of both of them are as follows. At the beginning, small initial spherulite with clear outline emerged and the birefringence showed a negative character. The growth rates $G$ of the initials spherulites in spherulite 1 and 2 were approximately 2.6 and $2.4 \mu \mathrm{m} / \mathrm{s}$ respectively, as shown in Figure 9. As the spherulite grew up, a new positive birefringence diffused on the edge of the spherulite, resulting in the formation of a relatively perfect spherulite with a double concentric feature. After that, the initial spherulite continued to grow and the growth rate remained constant. Before long, the initial spherulite stopped growing and its outline became indistinct. Moreover, the final sizes of both initial spherulites were similar (about $100 \mu \mathrm{m}$ ). At the same time, the outer birefringence pattern spread out with a much higher growth rate $(8.4 \mu \mathrm{m} / \mathrm{s}$ for spherulite 1 and $7.4 \mu \mathrm{m} / \mathrm{s}$ for spherulite 2) to cover 


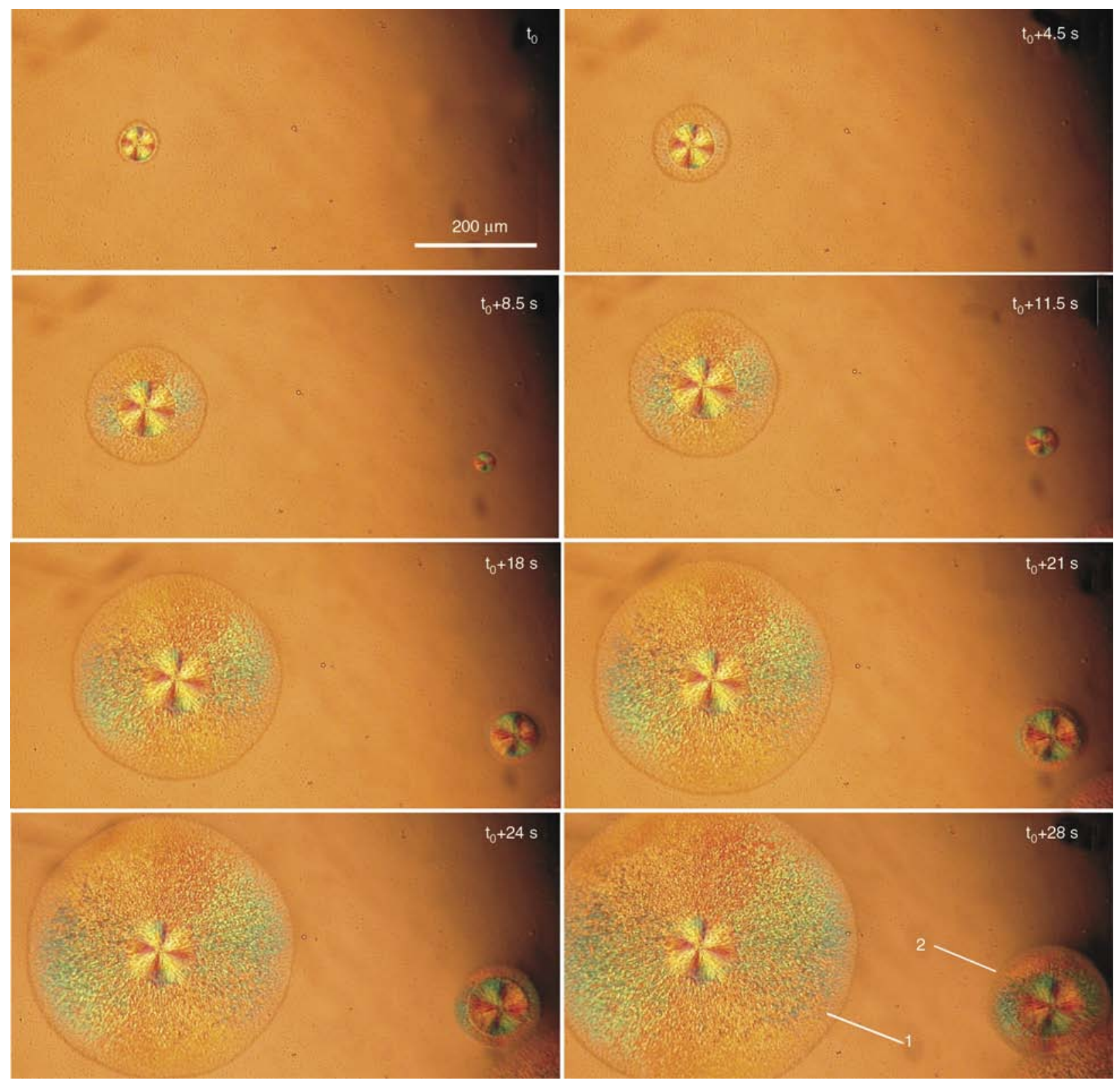

Figure 8. In situ POM micrographs of the isothermal crystallization of Block 7800 at $22^{\circ} \mathrm{C}$

all the remaining regions of sample. Finally, the whole birefringence was invariable, and the final texture of the double concentric spherulites was constructed.

The growth of double concentric spherulites was further investigated by time-dependent FTIR spectroscopy as presented in Figure 10. To ensure the accuracy, measurements were repeated at least 3 times with different areas of sample detected. Results of FTIR confirmed that the PTHF segments were totally amorphous because there is no characteristic absorption at $564 \mathrm{~cm}^{-1}$ and the absorption at $746 \mathrm{~cm}^{-1}$ is constant. This means that both inner and outer spherulites were formed by the crystallization of PEG segments. This point was well illustrated by the continuous increase of vibrational bands at $843 \mathrm{~cm}^{-1}$ during the whole crystallization process.

This morphology was further investigated by SEM, as shown in Figure 11. In the initial spherulites, interlocked fibrillar structures were formed and the orientation was roughly in accordance with the radial direction (see Figure 11a). While, at the boundary between the initial spherulite and the outer part, the reticular structure vanished quickly. Then a new morphology containing dot-like and worm-like microzones formed, and spread out to construct the outer part of double concentric spherulites (see Figure 11b and Figure 11c).

As it has been observed that the initial spherulites formed in Block 7800 at $22^{\circ} \mathrm{C}$ had a constant final size of about $100 \mu \mathrm{m}$ even if the nucleation times 


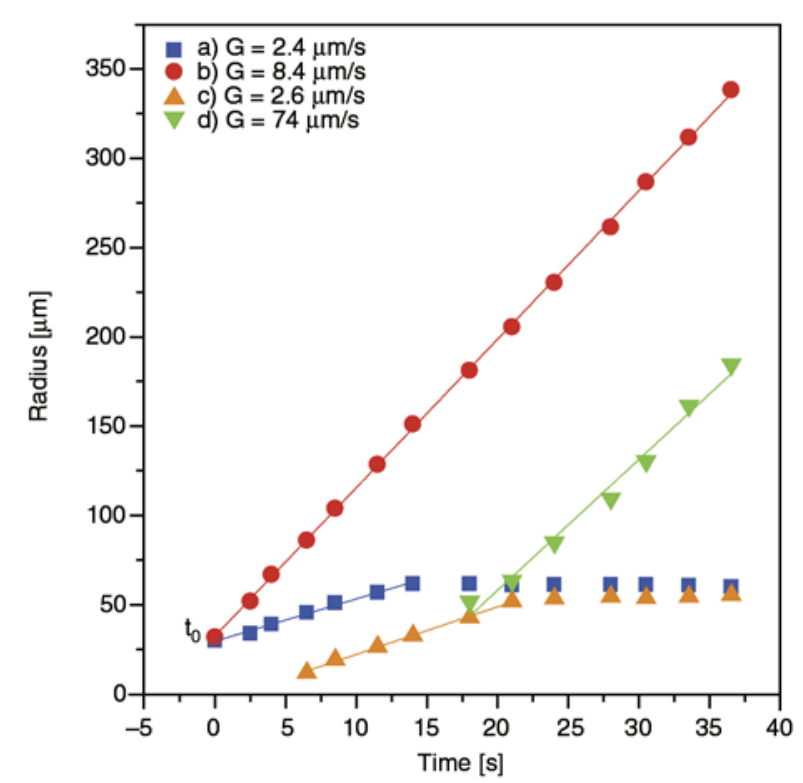

Figure 9. Spherulite radius plotted against the crystallization time at $22^{\circ} \mathrm{C}$ for Block7800. The slope of the straight line was taken as the radius growth rate G. (a) initial spherulite of spherulite 1. (b) outer part of spherulite 1. (c) initial spherulite of spherulite 2. (d) outer part of spherulite 2 .

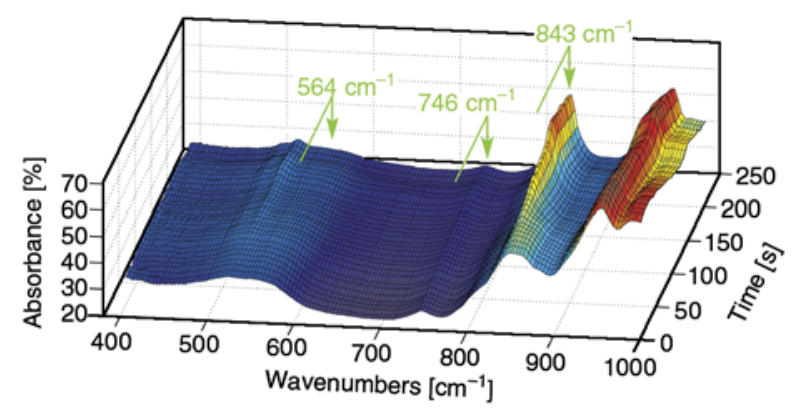

Figure 10. Time-dependent FTIR spectra of Block7800 at $22^{\circ} \mathrm{C}$

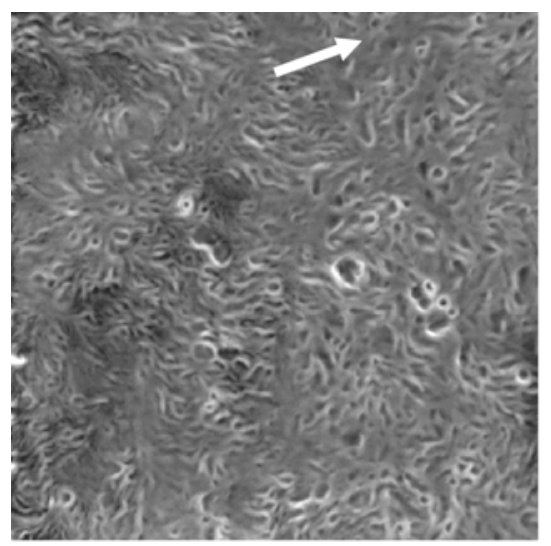

a)

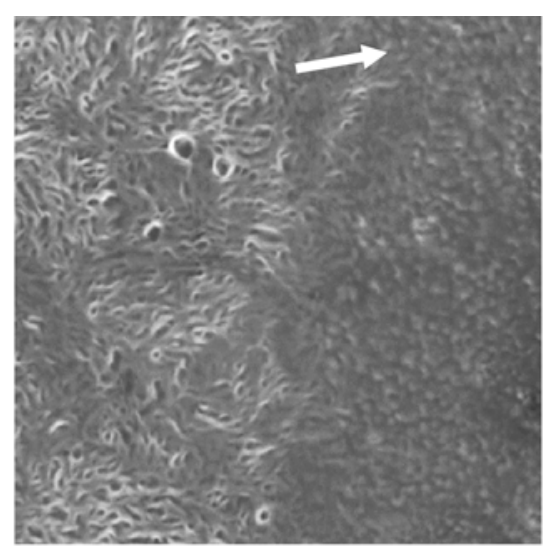

b) were different, we made an attempt to change the size of initial spherulite by adjusting the cooling rate during non-isothermal crystallization process. Morphologies of Block7800 with different cooling rates are illustrated in Figure 12. Unexpectedly, though the size of the outer part of double concentric spherulites increased dramatically as cooling rate slowed down, only feeble enlargement of initial spherulites was observed when the cooling rate dropped from 20 to $2^{\circ} \mathrm{C} / \mathrm{min}$. After size reach about $100 \mu \mathrm{m}$, the initial spherulites did not grow any more even with an extremely slow cooling rate of $0.1^{\circ} \mathrm{C} / \mathrm{min}$. Therefore, there was a size limit of initial spherulite. According to our observations in both isothermal crystallization processes with different temperatures and non-isothermal crystallization processes with different cooling rates, the maximum size was about $100 \mu \mathrm{m}$ in diameter.

Herein, our work reveals the fact that the crystals of semicrystalline ABA triblock copolymers are able to form double concentric spherulites under polarized-light microscopy. That is quite different from previous work [26-28], in which the morphologies of inner and outer parts of concentric spherulites are dominated by the crystallization of different segments of double crystalline block copolymer. This phenomena is confused and puzzling, but really interesting.

Though the triblock copolymer was prepared with high purity as mentioned above, we still suspected that the product contained traces of PEG diol. To find out whether the PEG diol was the cause of the formation of double concentric spherulites, we blended Block7800 with a small amount of PEG8000 and

Figure 11. Morphology in different regions of double concentric spherulites (formed at $4^{\circ} \mathrm{C}$ ) observed by SEM: (a) morphology of initial sperulite; (b) morphology at the boundary between the initial spherulite and outer part; (c) morphology of the outer part. The arrow indicates the radial direction from the center. 

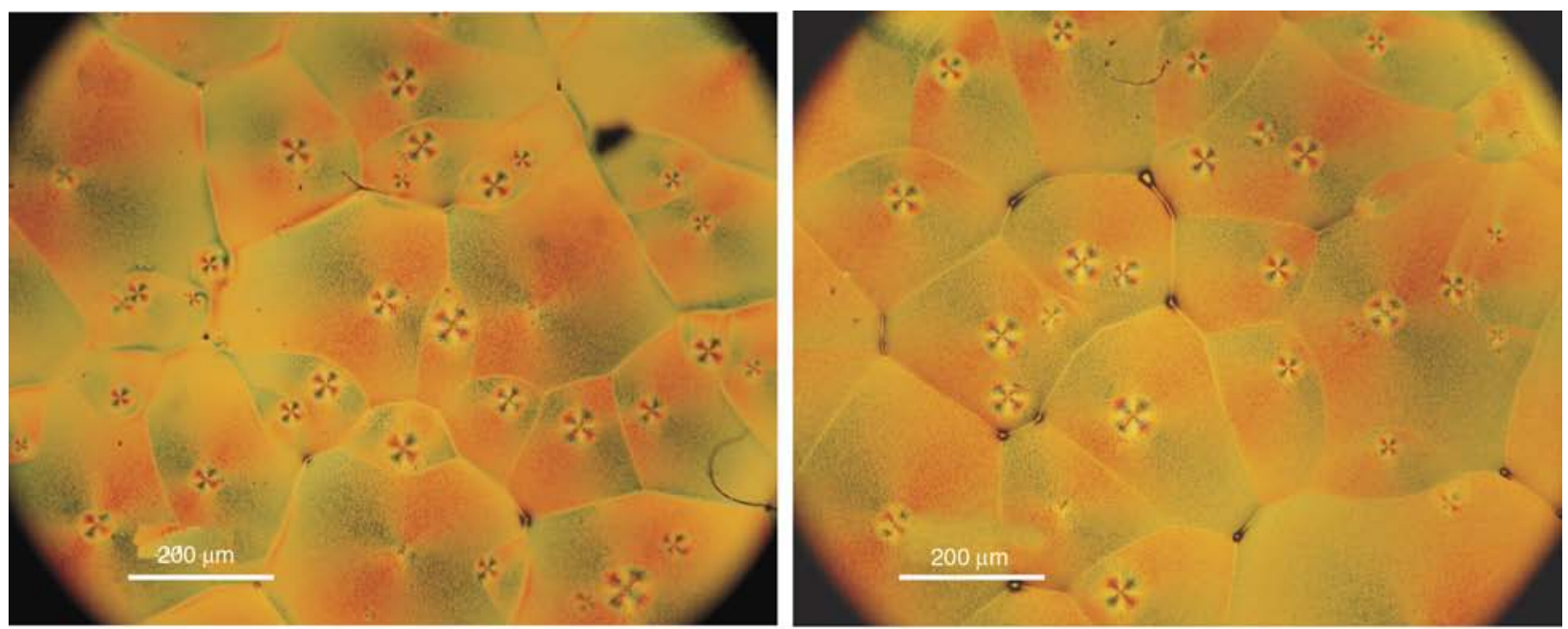

a)

b)

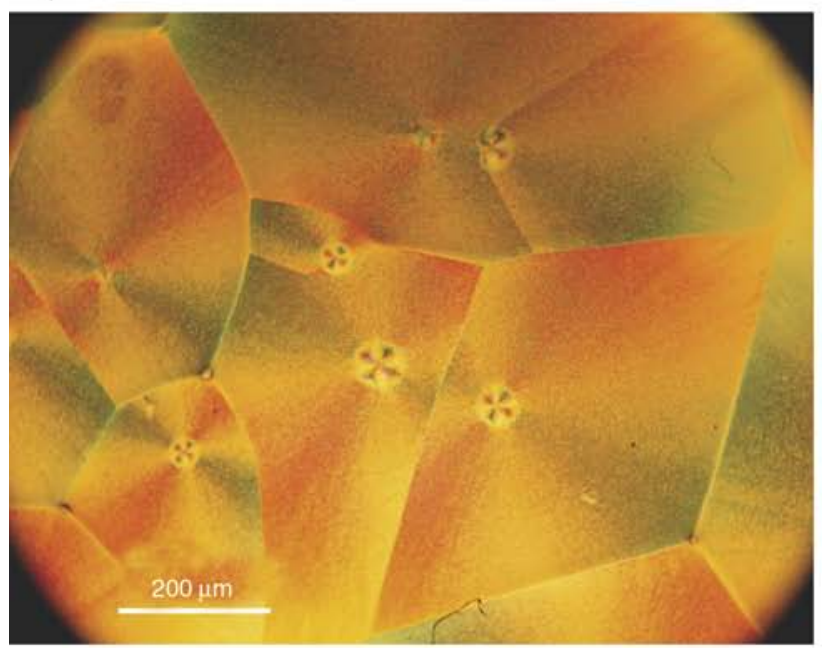

c)

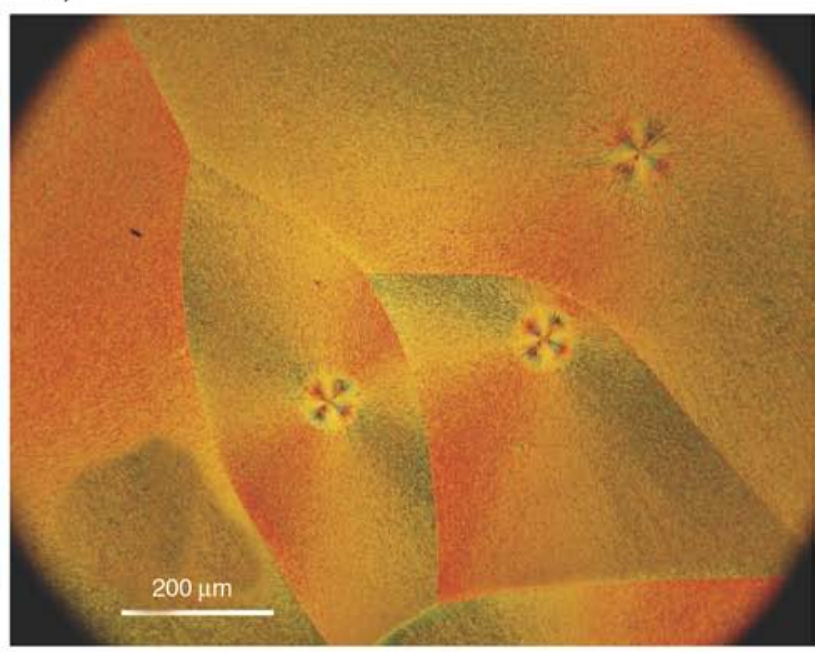

d)
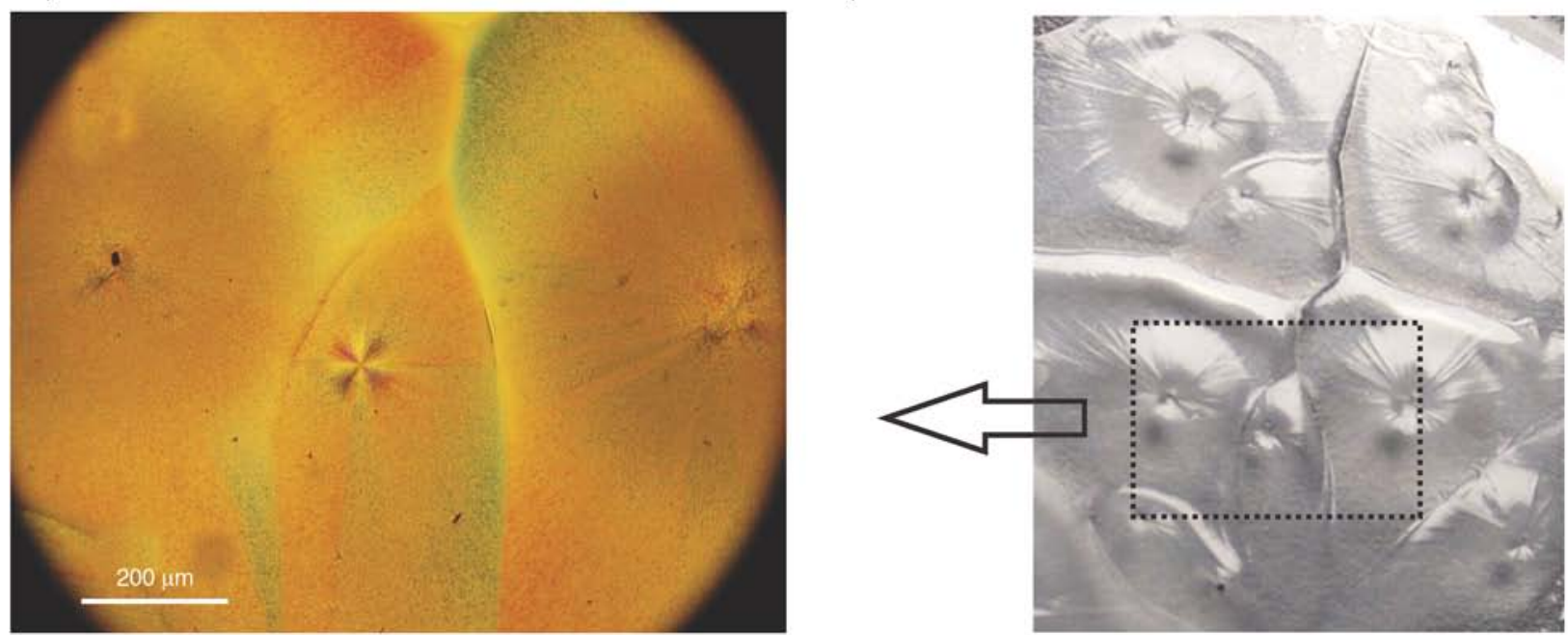

e)

f)

Figure 12. Morphologies of Block 7800 formed at different cooling rates: (a) $\left(20^{\circ} \mathrm{C} / \mathrm{min}\right)$, (b) $\left(10^{\circ} \mathrm{C} / \mathrm{min}\right),(\mathrm{c})\left(2^{\circ} \mathrm{C} / \mathrm{min}\right)$, (d) $\left(0.5^{\circ} \mathrm{C} / \mathrm{min}\right)$ and (e) $\left(0.1^{\circ} \mathrm{C} / \mathrm{min}\right)$ were taken by POM; (f) $\left(0.1^{\circ} \mathrm{C} / \mathrm{min}\right)$ was taken by camera with the micro distance photography pattern

PEG2000 (10 wt\%) respectively. The PEG diols added in had a similar molecular weight of Block 7800 or its PEG blocks. The morphologies are shown in Figure 13. As shown, the formation of double concentric spherulites was difficult in both blends. The initial spherulites were much smaller and hard to find. If the PEG diol caused the formation of double concentric spherulites, it should 


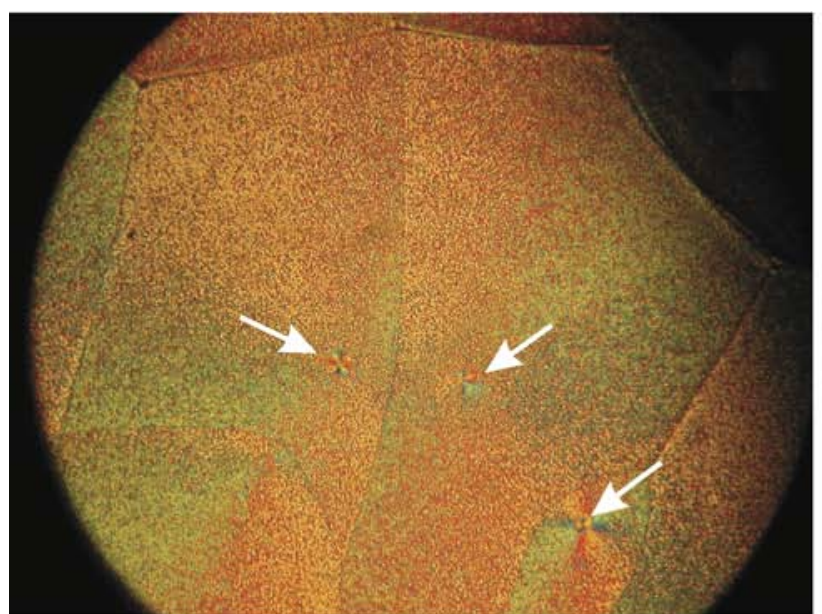

a)

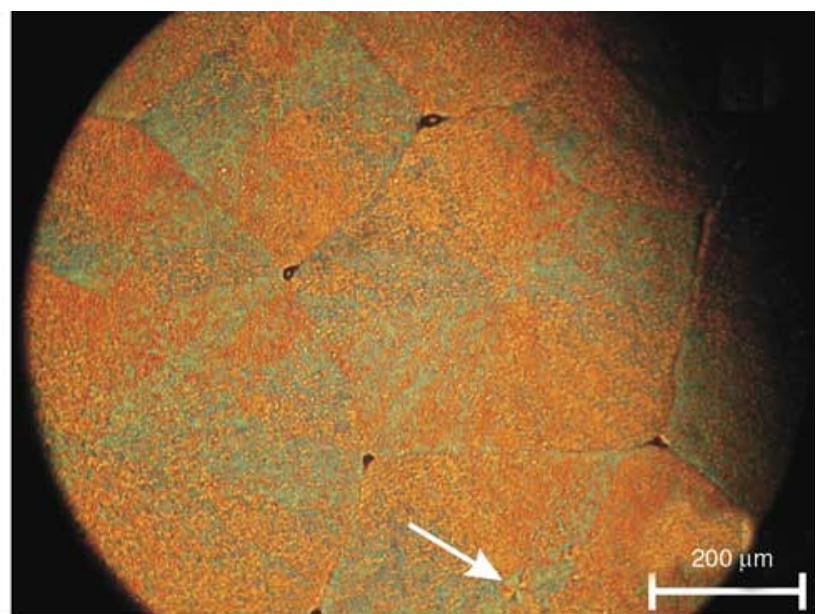

b)

Figure 13. POM micrographs: (a) Block7800 containing $10 \mathrm{wt} \%$ PEG2000; (b) Block7800 containing 10 wt $\%$ PEG8000. Polymer blends were melted at $80^{\circ} \mathrm{C}$ for $10 \mathrm{~min}$ and crystallized at $22^{\circ} \mathrm{C}$. The arrows point to the initial spherulites.

nucleate and crystallize earlier than block copolymer to form the initial spherulite, and then the number of the initial spherulite (or double concentric spherulites) would increase as PEG diol is added. This conjecture was denied by our observation. Hence the PEG diol was not the cause of the formation of double concentric spherulites.

Recently, Shi et al. [43] reported the concentric structure which was caused by the lamellar orientation inversion of PEG crystals due to the intervention of phase separation. When phase separation occurred, due to the dynamic competition between phase separation and crystallization process, PEG lamellas tended to array along different directions and formed different spherulitic morphology forms $[32,44]$. As Block7800 was proved to be crystalline-amorphous, the resulting crystal morphologies can be significantly influenced by microphase separation $[5,6]$. That may help to explain the formation of double concentric spherulites discovered in our study. Possible formation mechanism may be as follows. Firstly, the initial spherulites were formed and grew up, following the nucleation in polymer melt. After a certain period, phase separation occured. Then the PEG lamellar crystals were formed with the intervention of phase separation and adopted a different orientation, resulting in the formation of the outer part with a much different birefringence pattern. However, the detailed phase separation progress still needs to be intensively studied and the reason why the initial spherulite had a size limit has not been found out. Further investigation is in progress, and the results will be published elsewhere.

\section{Conclusions}

In this study, PEG-PTHF-PEG triblock copolymers with different molecular weights but the same block ratio were conveniently synthesized. The hydroxylended PTHF reacted with NaH firstly, and its sodium alcoholate was used as macroinitiator for anionic ring-opening polymerization of EO to obtain PEGPTHF-PEG triblock copolymer.

The molecular weights of triblock copolymers with the same block ratio exhibited a significant effect on their crystallization behavior, and even led to the transition of triblock copolymer from double crystalline to a crystalline-amorphous. At a low molecular weight of 2950, both PEG and PTHF blocks were crystallizable, and after the molecular weight of block copolymer increased to 4900, crystallization of PEG blocks became dominant. With the molecular weight increased to 7800 , only PEG blocks could crystallize and PTHF blocks were amorphous, where double concentric spherulites were formed. In addition, there is an upper limit for the size of the initial spherulite in double concentric spherulites.

\section{Acknowledgements}

This work was supported by the National Science Foundations of China (No. 21274116 and No. 21004049) and scientific research project of Ministry of Industry and Information Technology of P. R. China (No. J142011A001). 


\section{References}

[1] Hamley I. W.: Crystallization in block copolymers. Advances in Polymer Science, 148, 113-137 (1999). DOI: $10.1007 / 3-540-48836-72$

[2] Müller A. J., Balsamo V., Arnal M. L.: Nucleation and crystallization in diblock and triblock copolymers. Advances in Polymer Science, 190, 1-63 (2005).

DOI: $10.1007 / 12001$

[3] Nandan B., Hsu J-Y., Chen H-L.: Crystallization behavior of crystalline - Amorphous diblock copolymers consisting of a rubbery amorphous block. Journal of Macromolecular Science Part C: Polymer Reviews, 46, 143-172 (2006).

DOI: $10.1080 / 15321790600646802$

[4] Müller A. J., Arnal M. L., Balsamo V.: Crystallization in block copolymers with more than one crystallizable block. Lecture Notes in Physics, 714, 229-259 (2007). DOI: $10.1007 / 3-540-47307-613$

[5] Castillo R. V., Müller A. J.: Crystallization and morphology of biodegradable or biostable single and double crystalline block copolymers. Progress in Polymer Science, 34, 516-560 (2009).

DOI: 10.1016/j.progpolymsci.2009.03.002

[6] Shin D., Shin K., Aamer K. A., Tew G. N., Russell T. P., Lee J. H., Jho J. Y.: A morphological study of a semicrystalline poly(L-lactic acid- $b$-ethylene oxide- $b$ L-lactic acid) triblock copolymer. Macromolecules, 38, 104-109 (2005). DOI: $10.1021 / \mathrm{ma} 0481712$

[7] Hong S., MacKnight W. J., Russell T. P., Gido S. P.: Structural evolution of multilayered, crystalline-amorphous diblock copolymer thin films. Macromolecules, 34, 2876-2883 (2001).

DOI: $10.1021 / \mathrm{ma} 002032 \mathrm{c}$

[8] Sun L., Liu Y., Zhu L., Hsiao B. S., Avila-Orta C. A.: Pathway-dependent melting in a low-molecular-weight polyethylene-block-poly(ethylene oxide) diblock copolymer. Macromolecular Rapid Communications, 25, 853-857 (2004).

DOI: $10.1002 /$ marc. 200300320

[9] Lin M-C., Wang Y-C., Chen J-H., Chen H-L., Müller A. J., Su C. J., Jeng U-S.: Orthogonal crystal orientation in double-crystalline block copolymer. Macromolecules, 44, 6875-6884 (2011).

DOI: $10.1021 / \mathrm{ma} 201124 \mathrm{u}$

[10] Maglio G., Migliozzi A., Palumbo R.: Thermal properties of di- and triblock copolymers of poly(L-lactide) with poly(oxyethylene) or poly( $\varepsilon$-caprolactone). Polymer, 44, 369-375 (2003).

DOI: $10.1016 / \mathrm{S} 0032-3861(02) 00764-4$

[11] Sun L., Liu Y., Zhu L., Hsiao B. S., Avila-Orta C. A.: Self-assembly and crystallization behavior of a double-crystalline polyethylene-block-poly(ethylene oxide) diblock copolymer. Polymer, 45, 8181-8193 (2004). DOI: $10.1016 /$ j.polymer.2004.09.063
[12] Weiyu C., Tashiro K., Hanesaka M., Takeda S., Masunaga H., Sasaki S., Takata M.: Relationship between morphological change and crystalline phase transitions of polyethylene-poly(ethylene oxide) diblock copolymers, revealed by the temperature-dependent synchrotron WAXD/SAXS and infrared/raman spectral measurements. The Journal of Physical Chemistry B, 113, 2338-2346 (2009).

DOI: $10.1021 / \mathrm{jp} 8092435$

[13] Bogdanov B., Vidts A., Schacht E., Berghmans H.: Isothermal crystallization of poly( $\varepsilon$-caprolactone-ethylene glycol) block copolymers. Macromolecules, 32, 726-731 (1999).

DOI: $10.1021 / \mathrm{ma980226a}$

[14] Shiomi T., Imai K., Takenaka K., Takeshita H., Hayashi H., Tezuka Y.: Appearance of double spherulites like concentric circles for poly( $\varepsilon$-caprolactone)-block-poly (ethylene glycol)-block-poly( $\varepsilon$ caprolactone). Polymer, 42, 3233-3239 (2001).

DOI: $10.1016 / \mathrm{S} 0032-3861(00) 00597-8$

[15] Takeshita H., Fukumoto K., Ohnishi T., Ohkubo T., Miya M., Takenaka K., Shiomi T.: Formation of lamellar structure by competition in crystallization of both components for crystalline-crystalline block copolymers. Polymer, 47, 8210-8218 (2006).

DOI: $10.1016 /$ j.polymer.2006.09.043

[16] Nojima S., Fukagawa Y., Ikeda H.: Interactive crystallization of a strongly segregated double crystalline block copolymer with close crystallizable temperatures. Macromolecules, 42, 9515-9522 (2009).

DOI: $10.1021 / \mathrm{ma901964a}$

[17] Castillo R. V., Müller A. J., Raquez J-M., Dubois P.: Crystallization kinetics and morphology of biodegradable double crystalline PLLA- $b$-PCL diblock copolymers. Macromolecules, 43, 4149-4160 (2010). DOI: $10.1021 / \mathrm{ma100201 \textrm {g }}$

[18] Salmerón Sánchez M., Mathot V. B. F., Vanden Poel G., Gómez Ribelles J. L.: Effect of the cooling rate on the nucleation kinetics of poly(L-lactic acid) and its influence on morphology. Macromolecules, 40, 79897997 (2007). DOI: $10.1021 / \mathrm{ma} 0712706$

[19] Lee L-B. W., Register R. A.: Equilibrium control of crystal thickness and melting point through block copolymerization. Macromolecules, 37, 7278-7284 (2004). DOI: $10.1021 / \mathrm{ma} 049143 \mathrm{~m}$

[20] Li S., Myers S. B., Register R. A.: Solid-state structure and crystallization in double-crystalline diblock copolymers of linear polyethylene and hydrogenated polynorbornene. Macromolecules, 44, 8835-8844 (2011). DOI: $10.1021 / \mathrm{ma} 201951 \mathrm{j}$

[21] Sun J., Chen X., He C., Jing X.: Morphology and structure of single crystals of poly(ethylene glycol)poly( $\varepsilon$-caprolactone) diblock copolymers. Macromolecules, 39, 3717-3719 (2006).

DOI: $10.1021 / \mathrm{ma} 0603074$ 
[22] Hobbs J. K., Register R. A.: Imaging block copolymer crystallization in real time with the atomic force microscope. Macromolecules, 39, 703-710 (2006).

DOI: $10.1021 / \mathrm{ma} 0514020$

[23] Zhu L., Cheng S. Z. D., Calhoun B. H., Ge Q., Quirk R. P., Thomas E. L., Hsiao B. S., Yeh F., Lotz B.: Crystallization temperature-dependent crystal orientations within nanoscale confined lamellae of a self-assembled crystalline-amorphous diblock copolymer. Journal of the American Chemical Society, 122, 59575967 (2000).

DOI: $10.1021 / \mathrm{ja} 000275 \mathrm{e}$

[24] Li C. Y., Cheng S. Z. D., Ge J. J., Bai F., Zhang J. Z., Mann I. K., Chien L-C., Harris F. W., Lotz B.: Molecular orientations in flat-elongated and helical lamellar crystals of a main-chain nonracemic chiral polyester. Journal of the American Chemical Society, 122, 72-79 (1999).

DOI: $10.1021 /$ ja993249t

[25] Kong J., Fan X., Qiao W., Xie Y., Si Q., Tang Y.: Study of a skin-core type of crystallinity distribution within polyethylene specimen crystallized under high pressure. Polymer, 46, 7644-7651 (2005).

DOI: $10.1016 /$ j.polymer.2005.04.108

[26] He C., Sun J., Deng C., Zhao T., Deng M., Chen X., Jing X.: Study of the synthesis, crystallization, and morphology of poly(ethylene glycol)-poly(e-caprolactone) diblock copolymers. Biomacromolecules, 5, 2042-2047 (2004).

DOI: $10.1021 / \mathrm{bm} 049720 \mathrm{e}$

[27] He C., Sun J., Zhao T., Hong Z., Zhuang X., Chen X., Jing X.: Formation of a unique crystal morphology for the poly(ethylene glycol)-poly( $\varepsilon$-caprolactone) diblock copolymer. Biomacromolecules, 7, 252-258 (2006). DOI: $10.1021 / \mathrm{bm} 050627 \mathrm{f}$

[28] He C., Sun J., Ma J., Chen X., Jing X.: Composition dependence of the crystallization behavior and morphology of the poly(ethylene oxide)-poly( $\varepsilon$-caprolactone) diblock copolymer. Biomacromolecules, 7, 34823489 (2006).

DOI: $10.1021 / \mathrm{bm} 060578 \mathrm{~m}$

[29] Zhu W., Xie W., Tong X., Shen Z.: Amphiphilic biodegradable poly(CL- $b$-PEG- $b$-CL) triblock copolymers prepared by novel rare earth complex: Synthesis and crystallization properties. European Polymer Journal, 43, 3522-3530 (2007).

DOI: 10.1016/j.eurpolymj.2007.04.024

[30] Albuerne J., Márquez L., Müller A. J., Raquez J. M., Degée P., Dubois P., Castelletto V., Hamley I. W.: Nucleation and crystallization in double crystalline poly $(p-$ dioxanone)- $b$-poly( $\varepsilon$-caprolactone) diblock copolymers. Macromolecules, 36, 1633-1644 (2003).

DOI: $\underline{10.1021 / \mathrm{ma} 025766 \mathrm{t}}$
[31] Jiang S., He C., An L., Chen X., Jiang B.: Crystallization and ring-banded spherulite morphology of poly (ethylene oxide)-block-poly( $\varepsilon$-caprolactone) diblock copolymer. Macromolecular Chemistry and Physics, 205, 2229-2234 (2004).

DOI: $10.1002 / \mathrm{macp} .200400204$

[32] Shi W., Yang J., Zhang Y., Luo J., Liang Y., Han C. C.: Lamellar orientation inversion under dynamic interplay between crystallization and phase separation. Macromolecules, 45, 941-950 (2012).

DOI: $10.1021 / \mathrm{ma} 202046 \mathrm{c}$

[33] Hevus I., Kohut A., Voronov A.: Amphiphilic invertible polyurethanes: Synthesis and properties. Macromolecules, 43, 7488-7494 (2010).

DOI: $10.1021 / \mathrm{ma101175k}$

[34] Niu L., Nagarajan R., Guan F., Samuelson L. A., Kumar J.: Biocatalytic synthesis of multi-block copolymer composed of poly(tetrahydrofuran) and poly(ethylene oxide). Journal of Macromolecular Science Part A: Pure and Applied Chemistry, 43, 1975-1981 (2006). DOI: 10.1080/10916460600997744

[35] Ikeda T., Lee W. K., Ooya T., Yui N.: Thermodynamic analysis of inclusion complexation between $\alpha$-cyclodextrin-based molecular tube and poly(ethylene oxide)block-poly(tetrahydrofuran)-block-poly(ethylene oxide) triblock copolymer. The Journal of Physical Chemistry B, 107, 14-19 (2003). DOI: $10.1021 / j p 020817+$

[36] Pomel C., Leborgne C., Cheradame H., Scherman D., Kichler A., Guegan P.: Synthesis and evaluation of amphiphilic poly(tetrahydrofuran- $b$-ethylene oxide) copolymers for DNA delivery into skeletal muscle. Pharmaceutical Research, 25, 2963-2971 (2008). DOI: $10.1007 / \mathrm{s} 11095-008-9698-9$

[37] De Witte I. C., Goethals E. J.: Synthesis and block-specific complexation of poly(ethylene oxide)-poly(tetrahydrofuran)-poly(ethylene oxide) triblock copolymers. Polymers for Advanced Technologies, 10, 287-292 (1999).

DOI: 10.1002/(SICI)1099-1581(199905)10:5<287:: AID-PAT877>3.0.CO;2-F

[38] Libera M., Trzebicka B., Kowalczuk A., Wałach W., Dworak A.: Synthesis and thermoresponsive properties of four arm, amphiphilic poly(tert-butyl-glycidylether)-block-polyglycidol stars. Polymer, 52, 250-257 (2011).

DOI: $10.1016 /$ j.polymer.2010.12.001

[39] Feng X-S., Taton D., Chaikof E. L., Gnanou Y.: Toward an easy access to dendrimer-like poly(ethylene oxide)s. Journal of the American Chemical Society, 127, 10956-10966 (2005). DOI: $10.1021 / \mathrm{ja} 0509432$ 
[40] Müller A. J., Albuerne J., Marquez L., Raquez J-M., Degée P., Dubois P., Hobbs J., Hamley I. W.: Selfnucleation and crystallization kinetics of double crystalline $\operatorname{poly}(p$-dioxanone)- $b$-poly( $\varepsilon$-caprolactone) diblock copolymers. Faraday Discuss, 128, 231-252 (2005).

DOI: $10.1039 / \mathrm{b} 403085 \mathrm{k}$

[41] Delahaye N., Duclos R., Saiter J. M.: Characterization of PEG6000 phase transitions: Influence of kinetic conditions. International Journal of Pharmaceutics, 157, 27-34 (1997).

DOI: $10.1016 / \mathrm{S} 0378-5173(97) 00207-X$

[42] Yin J. H., Mo Z. S.: Modern polymer physics (in Chinese). Science Press, Beijing (2001).
[43] Shi W., Cheng H., Chen F., Liang Y., Xie X., Han C. C.: Concentric ring pattern formation in a competing crystallization and phase separation process. Macromolecular Rapid Communications, 32, 1886-1890 (2011).

DOI: $10.1002 /$ marc. 201100490

[44] Shi W., Han C. C.: Dynamic competition between crystallization and phase separation at the growth interface of a PMMA/PEO blend. Macromolecules, 45, 336-346 (2011).

DOI: $10.1021 / \mathrm{ma} 201940 \mathrm{~m}$ 